\title{
Diagnóstico e Tratamento das Lombalgias e Lombociatalgias(*)
}

\author{
Brazil AV, Ximenes AC, Radu AS, Fernades AR, Appel C, Maçaneiro CH, Ribeiro CH, Gomes C, Meirelles
} ES, Puertas EB, Landin E, Egypto EJP, Appel F, Dantas FLR, Façanha F FAM, Furtado GE, Carneiro F ${ }^{\circ}$ GS, Cecin HA, Defino HL, Carrete Jr. H, Natour J, Marques Neto JF, Amaral F JC, Provenza JR, Vasconcelos JTS, Amaral LLF, Vialle LRG, Masini M, Taricco MA, Brotto MWI, Daniel MM, Sposito M, Morais OJS, Botelho RV, Xavier RM, Radominski SC, Daher S, Lianza S, Amaral SR, Antonio SF, Barros $F^{\circ}$ TE, Viana U, Vieira VP, Ferreira WHR, Stump XMG

\section{DESCRIÇÃO DO MÉTODO DE COLETA DE EVIDENNCIAS}

Reunião consensual e multidisciplinar para elaboração do texto com inclusão das citações bibliográficas, numa colaboração das especialidades de reumatologia, ortopedia e traumatologia, neurocirurgia, radiologia, medicina física e reabilitação e patologia da coluna vertebral. A partir de um texto básico referencial elaborado pelo editor médico, os participantes, divididos em cinco grupos de trabalho, geraram, por acréscimos e subtrações ao texto básico, recomendações aprovadas, posteriormente, em plenária, que permitiram a edição de um texto preliminar. $\mathrm{O}$ documento do consenso foi veiculado pela Internet, para consulta pública, tendo recebido várias sugestões e comentários de especialistas no assunto. As propostas foram devidamente avaliadas por uma comissão julgadora e revisora, que selecionou as que foram incorporadas ao texto preliminar. $\mathrm{O}$ editor médico, a partir da versão revisada, chegou ao texto final publicado, que recebeu da Biblioteca Nacional o ISBN $\mathrm{n}^{\circ} 85-901548-1-5$. Uma versão resumida do referido con- senso, com algumas adequações, foi elaborada em trabalho colaborativo entre o editor médico e a comissão técnica do projeto diretrizes AMB/CFM.

\section{GRAU DE RECOMENDAÇÃO E FORÇA DE EVIDÊNCIA}

A: Grandes ensaios clínicos aleatorizados e meta-análises.

B: Estudos clínicos e observacionais bem desenhados.

C: Relatos e séries de casos clínicos.

D: Publicações baseadas em consensos ou opiniões de especialistas.

\section{OBJETIVOS}

Oferecer informações sobre o diagnóstico e tratamento das lombalgias e lombociatalgias.

\section{PROCEDIMENTOS}

Diagnósticos e terapêuticos para as lombalgias e lombociatalgias.

\section{INTRODUÇÃO}

A dor lombar constitui uma causa freqüente de morbidade e incapacidade, sendo sobrepujada apenas pela cefaléia na escala dos distúrbios dolorosos que afetam o homem. No entanto, quando do atendimento primário por médicos não-especialistas, para apenas $15 \%$ das lombalgias e lombociatalgias, se encontra uma causa específica ${ }^{(1)}(\mathbf{D})$.

As dificuldades do estudo e da abordagem das lombalgias e lombociatalgias decorrem de vários fatores, dentre os

\footnotetext{
* Trabalho realizado sob a coordenadoria e edição médica de Cecin HA, por representantes das seguintes sociedades médicas: Sociedade Brasileira de Reumatologia. Sociedade Brasileira de Ortopedia e Traumatologia. Sociedade Brasileira de Neurocirurgia. Colégio Brasileiro de Radiologia. Sociedade Brasileira de Medicina Física e Reabilitação. Elaboração final: 6 de junho de 2001.

O Projeto Diretrizes, iniciativa conjunta da Associação Médica Brasileira e Conselho Federal de Medicina, tem por objetivo conciliar informações da área médica a fim de padronizar condutas que auxiliem o raciocínio e a tomada de decisão do médico. As informações contidas neste projeto devem ser submetidas à avaliação e à crítica do médico, responsável pela conduta a ser seguida, frente à realidade e ao estado clínico de cada paciente a ser seguida, frente à realidade e ao estado clínico de cada paciente.
} 
quais, podem ser mencionados a inexistência de uma fidedigna correlação entre os achados clínicos e os de imagem $^{(2)}(\mathbf{B})$; ser o segmento lombar inervado por uma difusa e entrelaçada rede de nervos, tornando dificil determinar com precisão o local de origem da dor, exceto nos acometimentos radículo-medulares; pelo fato das contraturas musculares, freqüentes e dolorosas, não se acompanharem de lesão histológica demonstrável; e, por serem raramente cirúrgicas, há escassas e inadequadas informações quanto aos achados anatômicos e histológicos das estruturas possivelmente comprometidas, o que torna dificil a interpretação do fenômeno doloroso.

Tais fatos fazem da caracterização etiológica da síndrome dolorosa lombar um processo eminentemente clínico, onde os exames complementares devem ser solicitados apenas para confirmação da hipótese diagnóstica.

Do ponto de vista evolutivo, as lombalgias, lombociatalgias e ciáticas podem ser caracterizadas como agudas ou lumbagos, subagudas e crônicas ${ }^{(3)}(\mathrm{D})$.

As dores lombares podem ser primárias ou secundárias, com ou sem envolvimento neurológico ${ }^{(4)}(\mathbf{D})$. Por outro lado, afecções localizadas neste segmento, em estruturas adjacentes ou mesmo à distância, de natureza a mais diversa, como congênitas, neoplásicas, inflamatórias, infecciosas, metabólicas, traumáticas, degenerativas e funcionais, podem provocar dor lombar.

A lombalgia idiopática, antigamente assim chamada, pois não se achava um substrato para sua causa, e que hoje é denominada de lombalgia mecânica comum, ou lombalgia inespecífica, é a forma anatomoclínica inicial de apresentação e a mais prevalente das causas de natureza mecânicodegenerativa.

Inúmeras circunstâncias contribuem para o desencadeamento e cronificação das síndromes dolorosas lombares (algumas sem uma nítida comprovação de relação causal) tais como: psicossociais, insatisfação laboral ${ }^{(5)}(\mathbf{B})$, obesidade $^{(6)}(\mathbf{B})$, hábito de fumar ${ }^{(7)}(\mathbf{B})$, grau de escolaridade, realização de trabalhos pesados ${ }^{(8)}(\mathbf{B})$, sedentarismo, síndromes depressivas ${ }^{(9)}(\mathbf{B})$, litígios trabalhistas ${ }^{(10)}(\mathbf{B})$, fatores genéticos e antropológicos, hábitos posturais, alterações climáticas, modificações de pressão atmosférica e temperatura ${ }^{(11)}(\mathbf{B})$.

Condições emocionais podem levar à dor lombar ou agravar as queixas resultantes de outras causas orgânicas preexistentes $^{(12)}(\mathbf{B})$.

\section{DIAGNÓSTICO CLÍNICO}

OS ELEMENTOS DA ANAMNESE E SUA FISIOPATOLOGIA NORTEIAM O RACIOCÍNIO DIAGNÓSTICO QUANTO À:

Intensidade, horário de aparecimento e outras características da dor.

- Na lombalgia mecânica comum (a forma mais prevalente), na maioria dos casos, se limita à região lombar e nádegas. Raramente se irradia para as coxas. Pode aparecer subitamente pela manhã e apresentar-se acompanhada de escoliose antálgica. $\mathrm{O}$ episódio doloroso tem duração média de três a quatro dias. Após esse tempo, o paciente volta à completa normalidade, com ou sem tratamento.

- Na hérnia de disco, quando se realiza um esforço de flexão durante o dia, o material nuclear é impelido para trás, em sentido antero-posterior, através das fibras do anel fibroso, mas por ele ainda é contido. Neste momento pode ainda não aparecer dor. No entanto, durante a noite, em razão de uma maior embebição aquosa do núcleo e conseqüente elevação da pressão intradiscal, as fibras do anel se rompem, dando então início, durante as primeiras horas do dia, à sintomatologia de quadro doloroso agudo, intenso, com irradiação da dor para um ou outro membro inferior e com manobras semióticas positivas de compressão radicular. A dor se exacerba com os esforços ${ }^{(13)}(\mathbf{C})$.

- No osteoma osteóide, a dor é desencadeada pela liberação de prostaglandinas pelas células tumorais durante a madrugada. Os pacientes se queixam de dor neste período, ou no começo do dia ${ }^{(14)}(\mathrm{D})$.

- No estreitamento do canal raquidiano artrósico, a dor lombar, às vezes, é noturna; outras vezes, à ela se associa ciatalgia uni ou bilateral intensa, que melhora ao sentar-se. Pode ser acompanhada de dor na panturrilha e de claudicação neurogênica intermitente. O processo doloroso piora ao caminhar, principalmente ladeira abaixo, e melhora ladeira acima, o que a diferencia da claudicação vascular, que piora ladeira acima. O sinal de Lasègue é negativo, enquanto na hérnia discal pode ser positivo. A manobra de Romberg é positiva. A extensão da coluna lombar, durante 30 segundos, desencadeia a $\operatorname{dor}^{(15)}(\mathbf{B})$.

- Nas espondiloartropatias soronegativas, que são doenças reumáticas inflamatórias, é característica a exacerbação matinal dos sintomas; aqui, a fisiopatogenia da dor é influenciada pelo ritmo circadiano da secreção do cortisol e pelo sistema nervoso autônomo ${ }^{(16)}$ (D). A sacro-iliíte bilateral, às vezes unilateral, consolida o diagnóstico. $\mathrm{Na}$ espondilite anquilosante, a dor pode ter uma característica especial: uma pseudociatalgia alternante. Nesta doença, um conjunto 
de cinco informações, prestadas pelo paciente, que inclui lombalgia de caráter insidioso, antes dos quarenta anos de idade, com duração maior do que três meses, acompanhada de rigidez matinal e melhora com a atividade física, apresenta sensibilidade de $95 \%$ e especificidade de $85 \%$ para a sua identificação ${ }^{(17)}(\mathbf{D})$.

\section{RELAÇÃO EXISTENTE ENTRE A DOR E A ATIVIDADE CORPORAL OU REPOUSO}

Dor com o movimento corporal ao longo do dia, ou desencadeada por longos períodos de permanência em pé, pode ser devido à alterações mecânicas ou degenerativas.

\section{ASSOCIAÇÃO DA DOR COM QUEIXAS SISTÊMICAS}

Quando houver comprometimento sistêmico, a dor lombar, geralmente, tem um começo gradual e progressivo, distribuição simétrica ou alternante, sem relação com o movimento e sem melhora com o repouso, e pode ser acompanhada de rigidez matinal de duração superior a trinta minutos $^{(18)}(\mathbf{D})$.

\section{TIPO DE IRRADIAÇÃO DA DOR: DISTRIBUIÇÃO DERMATOMÉRICA OU NÃO}

Quando a dor se irradia para a face anterior da coxa, não ultrapassando o joelho, deve-se pensar em neuralgia $\operatorname{crural}^{(19)}(\mathbf{D})$.

\section{DOR DE ORIGEM RAQUIDIANA OU EXTRA-RAQUIDIANA}

A dor de origem extra-raquidiana não tem relação com os movimentos da coluna, aparecendo mesmo com o repouso. Nesta situação, devem ser lembradas a calculose renal, endometriose, aneurisma de aorta abdominal, processos expansivos abdominais, retroperitoniais e outros.

\section{DOR PSICOSSOMÁTICA}

Pode ser detectada em pacientes que apresentem sensibilidade dolorosa superficial ou de distribuição não-anatômica, com queixa de dor vaga, imprecisa, um dia num lugar, outro dia em outro, com irradiação bizarra para peito, coluna dorsal, abdomen e dramatização do quadro clínico $^{(12)}(\mathbf{B})$.

OS ELEMENTOS DO EXAME FÍSICO E A SUA

FISIOPATOLOGIA, FUNDAMENTAIS PARA O RACIOCÍNIO DIAGNÓSTICO, SÃO:

- Flexão e extensão da coluna lombar

$\mathrm{O}$ aumento da pressão intradiscal durante a flexão da coluna lombar impele o disco para trás, no sentido ântero- posterior, piorando a dor na hérnia de disco. Há melhora ao deitar, posição onde a pressão intradiscal vai quase a zero. No estreitamento artrósico do canal raquidiano a dor piora com a extensão(20) $(B)$.

- Manobra de Valsalva

Na compressão radicular a manobra provoca exacerbação da dor ou irradiação dela até o pé, que não acontecia antes $^{(21)}(\mathbf{D})$.

- Manobra de Lasègue

É geralmente considerada positiva quando a dor se irradia, ou se exarceba, no trajeto do dermátomo de $\mathrm{L}_{4}-\mathrm{L}_{5}$, ou $\mathrm{L}_{5}-\mathrm{S}_{1}$, quando a elevação do membro inferior faz um ângulo de $35^{\circ}$ a $70^{\circ}$ com o plano horizontal. Sua positividade a $60^{\circ}$ comprova compressão radicular ${ }^{(22)}(\mathbf{D})$.

- Manobra de Romberg

É considerada anormal, se o movimento compensatório do corpo for necessário para manter os pés fixos no mesmo lugar. Este sinal costuma ser positivo na estenose do canal $^{(15)}(B)$.

- Sinal das pontas

Não se consegue andar com um dos calcanhares: compressão da raiz $\mathrm{L}_{5}$.

Não se consegue andar com uma das pontas dos pés: compressão da raiz $\mathrm{S}_{1}{ }^{(23)}(\mathbf{D})$.

- Sinal do arco de corda

Levanta-se a perna do paciente, como na manobra de Lasègue, até que a dor apareça; nesse momento, faz-se uma flexão do joelho. Havendo redução e/ou desaparecimento da dor, o sinal é considerado positivo para o diagnóstico de hérnia discal ${ }^{(24)}(\mathbf{D})$.

- Sinais não-orgânicos de lombalgias psicossomáticas

Há simulação de dor lombar ao se fazer compressão axial no topo do crânio ou fazendo rotação da pelve e ombros, evitando movimentar a coluna, e discrepâncias no sinal de Lasègue, quando pesquisado sentado ou deitado ${ }^{(25)}$ (D).

- Sinais de alerta

Sinais ou sintomas apresentados pelo paciente que possam ser devidos a outras enfermidades sistêmicas que não à lombalgia aguda mecânica ${ }^{(26)}(\mathrm{D})$.

1. De tumor ou Infecção: idade acima de 50 ou abaixo de 20 ; história de câncer; sintomas como febre, calafrios, perda de peso, sem outra explicação convincente;

- Infecção bacteriana recente, dependentes químicos, imunossuprimidos;

dor com piora noturna;

dor com piora em decúbito dorsal. 
2. De fratura:

trauma maior; trauma menor em idosos ou osteoporóticos.

3. De síndrome de cauda eqüina: anestesia em sela; disfunção de bexiga; déficit neurológico progressivo ou grave em membros inferiores.

\section{DIAGNÓSTICO COMPLEMENTAR}

A tomografia computadorizada e a ressonância magnética têm indicação naquelas lombalgias e ciatalgias agudas que tenham evolução atípica e nas de evolução insatisfatória, cuja causa não foi determinada após seis semanas de tratamento clínico. A tomografia computadorizada é um método planar, segmentar, que permite boa avaliação dos desarranjos discais, das alterações degenerativas das faces intervertebrais (platôs vertebrais) e articulações zigapofisárias. Também avalia o canal vertebral, recessos laterais e forames intervertebrais. A sua boa resolução espacial permite melhor definição dos contornos ósseos. A ressonância magnética é método multiplanar que não utiliza radiação ionizante e com amplo campo de visão. Permite boa avaliação dos desarranjos discais e das alterações degenerativas. É particularmente útil na análise do conteúdo do canal vertebral, incluindo cone medular, raízes da cauda eqüina e medula óssea ${ }^{(27)}(D)$.

A eletroneuromiografia não está indicada nas lombalgias agudas e crônicas e nas lombociatalgias agudas. É o único método que produz informações sobre a fisiologia da raiz nervosa envolvida, ajudando a compor a relevância clínica, sendo, entretanto, fundamental no diagnóstico diferencial das outras doenças do sistema nervoso periférico que possam mimetizar um quadro radicular ${ }^{(28)}(\mathbf{B})^{(29)}(\mathbf{D})$.

A densitometria óssea não está indicada nas lombalgias mecânicas ou não, agudas ou não, como método de investigação inicial, podendo ser útil naqueles casos em que o RX simples mostra a presença de deformidade vertebral, do tipo colapso, ou osteopenia radiológica. Neste aspecto, o simples achado de perda de massa óssea, revelado por este exame, não indica que a osteoporose justifique a dor lombar. O médico deve estar alerta também para as várias situações clínicas de osteoporose secundária, nas quais o exame pode ser indicado, como no uso prolongado de corticosteróides, hiperparatireoidismo, etc. ${ }^{(30)}(\mathbf{D})$.

\section{TRATAMENTO CONSERVADOR}

\section{REPOUSO}

O repouso é eficaz tanto nas lombalgias, como nas lombociatalgias e ciáticas. Ele não pode ser muito prolongado, pois a inatividade tem também a sua ação deletéria sobre o aparelho locomotor. Assim que a atividade e a deambulação forem possíveis, o tempo de repouso pode ser encurtado e o paciente deve ser estimulado a retornar às suas atividades habituais, o mais rapidamente possível. Este aconselhamento resulta em retorno mais rápido ao trabalho, menor limitação funcional a longo prazo e menor taxa de recorrência ${ }^{(31,32)}(\mathbf{A})$.

O posicionamento em repouso, principalmente nas hérnias discais, geralmente é feito com o corpo em decúbito supino, com joelhos fletidos e pés apoiados sobre o leito e/ou com flexão das pernas num ângulo de $90^{\circ} \mathrm{com}$ as coxas e, um mesmo ângulo destas com a bacia, objetivando a retificação da coluna lombar (posição de Zassirchon). Nestas posições, ele reduz de forma expressiva a pressão sobre os discos intervertebrais e a musculatura paravertebral lombar. A sua duração é variável, dependendo do tipo da doença e da intensidade da dor. Em média, deve ser de três a quatro dias e, no máximo, de cinco a seis dias ${ }^{(33)}(\mathbf{D})$. Nos casos em que a dor continua intensa, os movimentos e a deambulação difíceis, ele pode ser prolongado, pois cada caso é um caso $^{(22)}$ (D).

\section{MEDICAMENTOS}

O tratamento medicamentoso das lombalgias e lombociatalgias, após afastadas causas específicas como neoplasias, fraturas, doenças infecciosas e inflamatórias, deve ser centrado no controle sintomático da dor para propiciar a recuperação funcional, o mais rapidamente possível ${ }^{(34)}(D)$.

Acetaminofen (paracetamol) na dose de $500 \mathrm{mg}, 4$ a 6 vezes ao dia, é eficaz na dor de intensidade discreta e moderada. O risco da utilização do medicamento é considerado baixo, mas deve ser usado com cautela em hepatopatas e pacientes em uso concomitante de antiinflamatórios ${ }^{(35)}(\mathbf{A})$.

Dipirona é utilizada com freqüência no nosso meio, na dose de $500 \mathrm{mg}$, até 4 vezes ao $\operatorname{dia}^{(36)}(\mathbf{A})$. Outros analgésicos disponíveis no mercado: ácido acetilsalicílico, clonixinato de lisina; viminol, flupirtina.

Os opióides não são recomendados na lombalgia crônica, pelo risco da dependência química; quando usados por tempo prolongado. São uma opção no tratamento da lombalgia e ciatalgia agudas e em casos muito restritos. $\mathrm{O}$ fosfato de codeína na dose de $30 \mathrm{mg}, 3$ a 4 vezes ao dia, tem 
como principais efeitos adversos, sonolência, déficit de atenção e constipação intestinal ${ }^{(37)}(\mathbf{A})$. O cloridrato de tramadol é usado na dose de $100 \mathrm{mg}$ a $400 \mathrm{mg}$ diários e tem os mesmos efeitos acima.

Antiinflamatórios não-hormonais (AINHs), na prática clínica, são os medicamentos mais empregados. Dependendo da dose utilizada, a intervalos regulares, têm efeitos analgésicos e antiinflamatórios ${ }^{(34,38)}$ (D). Todas as classes de antiinflamatórios podem ser úteis no tratamento da lombalgia, desde que usadas com precaução em pacientes de risco como os idosos. Os efeitos adversos destes medicamentos podem causar sérios problemas para o paciente, devendose considerar na sua escolha, a tolerabilidade e segurança, assim como a sua interação com outros medicamentos ${ }^{(39)}(\mathbf{A})$.

Corticoesteróides. Os resultados dos estudos controlados e randomizados sobre a eficácia dos corticoesteróides na lombalgia aguda mecânica ou nas não-mecânicas, seja por via parenteral ou epidural, são conflitantes ${ }^{(40)}(D)$. No entanto, na hérnia discal, considerando que a compressão radicular pode se acompanhar de inflamação, lesão axonal e das células de Schwann, a sua utilização pode oferecer vantagens adicionais, uma vez que a inibição do referido processo inflamatório é mais completa e eficaz do que com AINHs ${ }^{(38)}(D)$. A infiltração epidural com glicocorticóides ${ }^{(4)}(\mathbf{D})$, anestésicos ${ }^{(42)}(\mathbf{A})$ e opióides é uma opção no manejo da dor radicular aguda após falha com o tratamento conservador.

Relaxantes musculares como carisoprodol, ciclobenzaprina são também uma opção no tratamento, a curto prazo, da lombalgia aguda, demonstrando eficácia superior ao placebo. O uso, em associação com outros analgésicos e antiinflamatórios, traz benefícios adicionais no alívio da $\operatorname{dor}^{(43)}(\mathbf{B})$. Complicações potenciais incluem sonolência, tontura e constipação intestinal. Utilização prolongada não é recomendada.

Os benzodiazepínicos não parecem úteis e não estão indicados na lombalgia mecânica $\operatorname{comum}^{(44)}(\mathbf{D})$. Os antidepressivos não são recomendados na lombalgia mecânica aguda. Os antidepressivos tricíclicos são uma opção nas lombalgias crônicas, mesmo quando não associadas à depressão.

Calcitonina é recomendada apenas nos casos de fratura osteoporótica recente com componente doloroso e nas dores ósseas das metástases e doença de $\operatorname{Paget}^{(45)}(\mathbf{D})$.

\section{TRATAMENTO CIRÚRGICO}

A lombalgia mecânica comum é sempre de tratamento conservador. Se resistente e existindo um evidente substrato clínico-patológico para essa evolução atípica, podem ser feitas infiltrações nas discopatias (Modic tipo I, II ou III), infiltração de pontos dolorosos, infiltração perifacetária, denervação facetária e artrose do segmento vertebral ${ }^{(46)}$ (D).

O tratamento cirúrgico da hérnia discal está indicado nos casos com déficit neurológico grave agudo (menos de 3 semanas), com ou sem dor; na lombociatalgia hiperálgica e, nas outras de menor intensidade, apenas para os pacientes que não melhoram após 90 dias de adequado tratamento clínico. Na síndrome da cauda equiina (alteração de esfincter, potência sexual e paresia dos membros inferiores) a cirurgia está indicada em caráter emergencial, como também, nas lombalgias infecciosas (espondiodiscites) com evolução desfavorável ${ }^{(47)}(\mathbf{B})^{(48)}(\mathbf{A})$.

A indicação de cirurgia no canal lombar estreito é feita em caráter individual, caso a caso, na síndrome da cauda equiina (paresia de MMII, disfunção urinária e sexual); na claudicação neurogênica intermitente incapacitante e progressiva e na radiculopatia unilateral que não responde ao tratamento conservador ${ }^{(49,50)}(\mathbf{B})$.

A cirurgia também está indicada: na espondilolise, com espondilolistese, e espondilolistese degenerativa, com dor lombar que não melhora com tratamento clínico; escorregamento vertebral progressivo no jovem (mesmo assintomático); lombociatalgia e claudicação neurogênica devidas a canal estreito que não responderam ao protocolo de tratamento conservador ${ }^{(51)}(\mathbf{B})$.

\section{REABILITAÇÃO}

Os meios físicos de tratamento (frio e calor nas diversas modalidades) são meros coadjuvantes no processo de reabilitação. Não atuam sobre as causas e sobre a história natural das síndromes dolorosas lombares ${ }^{(52,53)}(\mathbf{D})$.

Em relação à estimulação elétrica transcutânea (Tens) existem controvérsias sobre sua real eficácia. Não está indicada como medida inicial na lombalgia mecânica aguda $^{(54)}(\mathbf{A})$.

Não existem evidências científicas que comprovem o benefício da acupuntura em pacientes lombálgicos, porque os resultados das pesquisas não são controlados para os fatores de confusão devido ao tamanho da amostra, do desenho do estudo e o uso de placebos ${ }^{(38)}(\mathbf{D})$.

Os exercícios aeróbicos e de fortalecimento da musculatura paravertebral são comprovadamente eficazes ${ }^{(55)}(\mathbf{D})$.

Órteses e tração vertebral necessitam de comprovação através de estudos prospectivos, controlados e randomizados, de melhor qualidade e consistência metodológica ${ }^{(56)}(\mathbf{A})$. 
A manipulação somente deve ser realizada em casos específicos e por médicos capacitados para tal procedimento ${ }^{(57)}(\mathrm{D})$.

A educação e o esclarecimento dos pacientes são fundamentais para a sua reabilitação. Estudos de meta-análise demostram evidências de que as "Escolas de Coluna" têm a curto prazo melhores resultados que as outras formas de tratamento. Existem ainda moderadas evidências que as "Escolas de Coluna" em lombalgias por problemas ocupa-

\section{REFERÊNCIAS}

1. Deyo RA, Phillips WR. Low back pain. A primary care challenge. Spine 21: 2826-32, 1996.

2. Cecin HA. Proposição de uma reserva anatomofuncional, no canal raquidiano, como fator interferente na fisiopatologia das lombalgias e lombociatalgias mecânico-degenerativas. Rev Assoc Med Bras 43:295-310, 1997.

3. Nachemson AL. Newest knowledge of low back pain. A critical look. Clin Orthop 279:8-20, 1992.

4. Sheon RP, et al. Soft tissue rheumatic pain. 3rd ed; 1996. p. 391.

5. Bigos SJ, Battie MC, Spengler DM, et al. A prospective study of work perceptions and psychosocial factors affecting the report of back injury. Spine 16:1-6, 1991.

6. Leboeuf-Y de C, Kyvik KO, Bruun NH. Low back pain and lifestyle. Part II. Obesity. Information from a population-based sample of 29 , 424 twin subjects. Spine 24:779-83, 1999.

7. Deyo RA, Bass JE. Lifestyle and low-back pain. The influence of smoking and obesity. Spine 14:501-6, 1989.

8. Cecin HA. et al. Dor lombar e trabalho pesado: aspectos epidemiológicos. Rev Bras Reumatol 32:157-62, 1992.

9. Leino P, Magni G. Depressive and distress symptoms as predictors of low backpain, neck-shoulder pain, and other musculoskeletal morbidity: a 10 years follow-up of metal industry employees. Pain 53:89-94, 1993

10. Heliovaara M, Makela M, Knekt P, et al. Determinants of sciatica and low-back pain. Spine 16:608-14, 1991

11. McGorry RW, Hsiang SM, Snook SH, et al. Meteorological conditions and self-report of low back pain. Spine 23:2096-102, 1998.

12. Kummel BM. Nonorganic signs of significance in low back pain. Spine 21:1077-81, 1996.

13. Adams MA, Dolan P, Hutton WC, et al. Diurnal changes in spinal mechanics and their clinical significance. J Bone Joint Surg $\mathrm{Br} 72$ : 266-70, 1990.

14. Renier JC, Bregon CH. Lombalgies. In: Encycl Med Chirur Paris: Appareil Loccomoteur, 1984. 15840 B10, 3

15. Katz JN, Dalgas M, Stucki G, et al. Degenerative lumbar spinal stenosis. Diagnostic value of history and physical examination. Arthritis Rheum 38:1236-41, 1995

16. Greenspan FS. Basic and clinical endocrinology. 5.ed. Connecticut: Aptleton and Lange; 1997.

17. Calin A, Porta J, Fries JF, et al. Clinical history as a screening test for ankylosing spondylitis. JAMA 237:2613-4, 1977

18. Jenner JR, Barry M. ABC of rheumatology. Low back pain. $\mathrm{Br}$ Med J 310:929-32, 1995 cionais são mais efetivas que recursos placebo ou que deixar os pacientes em lista de espera ${ }^{(58)}(\mathbf{A})$.

Métodos de tratamento, sem comprovação científica, podem representar perda de tempo e prejuízo financeiro, quando não, riscos à saúde dos pacientes. Neste aspecto, sempre é bom lembrar do preceito de Loeb: "Não faça ao paciente aquilo que não gostaria que fizessem com você mesmo"(59)(D).

19. Ecker A, Woltman WH. Meralgia paraestésica. JAMA 110:1650-2, 1938.

20. Adams MA, May S, Freeman BJ, et al. Effects of backward bending on lumbar intervertebral discs. Relevance to physical therapy treatments for low back pain. Spine 25:431-7, discussion 438, 2000.

21. Scham SM, Taylor TK. Tension signs in lumbar disc prolapse. Clin Orthop 75:195-204, 1971.

22. Wiesel SW et al. The lumbar spine. 2nd ed. Philadelphia: W.B. Saunders; 1996. p. 13

23. DeSèze S. Congresso Brasileiro de Reumatologia, 8, 1970, Recife. Anais.

24. Anderson GB, Deyo RA. History and physical examination in patients with herniated lumbar discs. Spine 21:10S-18S, 1996.

25. Waddell G, McCulloch JA, Kummel E, et al. Nonorganic physical signs in low back pain. Spine 5:117-25, 1980.

26. Bigos SJ, et al. Acute low back problems in adults. Agency for Health Care Policy and Research 95-0643, 1994.

27. Boos N, Lander PH. Clinical efficacy of imaging modalities in the diagnosis of low-back pain disorders. Eur Spine J 5:2-22, 1996.

28. Nardin RA, Patel MR, Gudas TF. EMG and MRI in the evaluation of radiculopathy. Muscle Nerve 22:151-5, 1999.

29. Willborn AJ, Aminoff MJ. AAEE Minimonograph \#32. The electrophysiologic examination in patients with radiculopathies. Muscle Nerve 11:1099-114,1988.

30. Prevention and management of osteoporosis. Consensus statement from the scientific advisory board of the osteoporosis society of canada. Can Med Assoc J 155:924-9, 1996.

31. Vroomen PC, de Krom MC, Wilmink JT, et al. Lack of effectiveness of bed rest for sciatica. N Engl J Med 340:418-23, 1999.

32. Waddell G, Feder G, Lewis M. Systematic reviews of bed rest and advice to stay active for acute low back pain. Br J Gen Pract 47: 647-52, 1997.

33. Nachemson AL. Newest knowledge of low back pain. A critical look. Clin Orthop 279:8-20, 1992.

34. Waddell G. Treatment: scientific evidence. In: Waddell G, editor. Edinburg: Churchil Livingstore 16:263-74,1998.

35. Hickey RF. Chronic low back pain: a comparison of diflunisal with paracetamol. N Z Med J 95:312-4, 1982.

36. Babej-Dolle R, Freytag S, Eckmeyer J, et al. Parenteral dipyrone versus diclofenac and placebo in patients with acute lumbago or sciatic pain: randomized observer-blind multicenter study. Int J Clin Pharmacol Ther 32:204-9, 1994.

37. Innes GD, Croskerry P, Worthington J. Ketorolac versus acetaminophen-codeine in the emergency department treatment of acute low back pain. J Emerg Med 16:49-56, 1998. 
38. Malanga GA, Nadler SF. Nonoperative treatment of low back pain. Mayo Clin Proc 74:1135-48, 1999.

39. Berry H, Bloom B, Hamilton EB. Naproxen sodium, diflunisal, and placebo in the treatment of chronic back pain. Ann Rheum Dis 41:129-32, 1982.

40. Nachemson A, Jonsson E, editors. Neck and back pain. Philadelphia: Lippinicott Williams \& Wilkins; 2000.

41. Spaccarelli KC. Lumbar and caudal epidural corticosteroid injections. Mayo Clin Proc 71:169-78, 1996.

42. Kaplan M, Dreyfuss P, Halbrook B, et al. The ability of lumbar medial branch blocks to anesthetize the zygapophysial joint. A physiologic challange. Spine 23:1847-52, 1998.

43. Basmajian JV. Acute back pain and spasm. A controlled multicenter trial of combined analgesic and antispasm agents. Spine 14:438-9, 1989.

44. Tan JC. Practical manual of physical medicine and reabilitation: diagnostics, therapeutics and basic problems. St. Louis: Mosby; 1998. p. $133-155 / 321-85$.

45. Borenstein DG. Low back pain: medical diagnosis and comprehensive management. 2 nd ed. Philadelphia: W.B. Saunders; 1995.

46. Jayson AMIV. Assesment and management of chronic back pain. Rev Bras Reumatol 36:251, 1996.

47. Abramovitz JN, Neff SR. Lumbar disc surgery: results of prospective lumbar discectomy study of the joint section on disorders of the spine peripheral nerves of the American Association of Neurological Surgeon and the Congress of Neurological Surgeons. Neurosurgery 29:301-7, discussion 307-8, 1991.

48. Gibson JN, Grant IC, Waddell G, et al. The Cochrane review of surgery for lumbar disc prolapse and degenerative lumbar spondylosis. Spine 24:1820-32, 1999.
49. Amundsen $\mathrm{T}$, Weber $\mathrm{H}$, Nordal HJ, et al. Lumbar spinal stenosis: conservative or surgical management? A prospective 10-year study. Spine 25:1424-35, discussiion 1435-6, 2000.

50. Lehto MU, Honkanen P. Factors influencing the outcome of operative treatment for lumbar spinal stenosis. Acta Neurochir 137:25-8, 1995.

51. Feffer HL, Wiesel SW, Cuckler JM, et al. Degenerative spondylolisthesis. To fuse or not to fuse. Spine10:287-9, 1985.

52. Michels E. Measurements in physical therapy. Phys Ther 63:153-8, 1989.

53. Borenstein D. Epidemiology, etiology, diagnostic evaluation, and treatment of low back pain. Curr Opin Rheumatol 8:124-9, 1996.

54. Deyo RA, Walsh NE, Martin DC, et al. A controlled trial of transcutaneous electrical nerve stimulation (TENS) and exercise for chronic low back pain. N Engl J Med 322:1627-34, 1990.

55. Liemohn W. Exercise and arthritis. Exercise and the back. Rheum Dis Clin North Am 16:945-70, 1990.

56. Beurskens AJ, de Vet HC, Koke AJ, et al. Efficacy of traction for nonspecific low back pain: 12 -week and 6-month results of a randomized clinical trial. Spine 22:2756-62, 1997.

57. Arkuzewski $Z$. The efficacy of manual treatment in low back pain: a clinical trial. Manual Med 2:68-71, 1986.

58. Van Tulder MW, Esmail R, Bombardier C, et al. Back schools for non: especific low back pain. Cochrane Reviews, 2000.

59. Cecin HA, et al. Reflexões sobre a eficácia do tratamento fisiátrico na osteoartrose. Rev Bras Reumatol 35:270-8, 1995. 